\title{
Network Intrusion detection using Oversampling technique and Machine learning algorithms
}

\author{
Hafiza Anisa Ahmed $^{\text {Corresp., } 1}$, Anum Hameed ${ }^{1}$, Narmeen Zakaria Bawany ${ }^{1}$ \\ ${ }^{1}$ Department of Computer Science and Software Engineering, Jinnah University for Women, Karachi, Sindh, Pakistan \\ Corresponding Author: Hafiza Anisa Ahmed \\ Email address: hafizaanisaahmed@gmail.com
}

The expeditious growth of the World Wide Web and the rampant flow of network traffic have resulted in a continuous increase of network security threats. Cyber attackers seek to exploit vulnerabilities in network architecture to steal valuable information or disrupt computer resources. Network Intrusion Detection System (NIDS) is used to effectively detect various attacks, thus providing timely protection to network resources from these attacks. To implement NIDS, stream of supervised and unsupervised machine learning approaches is applied to detect irregularities in network traffic and to address network security issues. Such NIDSs are trained using various datasets that include attack traces. However, due to the advancement in modern-day attacks, these systems are unable to detect the emerging threats. Therefore, NIDS needs to be trained and developed with a modern comprehensive dataset which contains contemporary common and attack activities. This paper presents a framework in which different machine learning classification schemes are employed to detect various types of network attack categories. Five machine learning algorithms: Random Forest, Decision Tree, Logistic Regression, KNearest Neighbors and Artificial Neural Networks, are used for attack detection. This study uses a dataset published by the University of New South Wales (UNSW-NB15), a relatively new dataset that contains a large amount of network traffic data with nine categories of network attacks. The results show that the classification models achieved the highest accuracy of $89.29 \%$ by applying the Random Forest algorithm. Further improvement in the accuracy of classification models is observed when Synthetic Minority Oversampling Technique (SMOTE) is applied to address the class imbalance problem. After applying the SMOTE, the Random Forest classifier showed an accuracy of $95.1 \%$ with 24 selected features from the Principal Component Analysis method. 


\section{Network Intrusion detection using Oversampling}

3 technique and Machine learning algorithms

Corresponding Author:

Hafiza Anisa Ahmed ${ }^{1}$, Anum Hameed ${ }^{1}$, Narmeen Zakaria Bawany ${ }^{1}$

${ }^{1}$ Department of Computer Science and Software Engineering, Jinnah University for Women, Karachi, Sindh, Pakistan

Hafiza Anisa Ahmed ${ }^{1}$

5C, Nazimabad, Karachi - 74600, Pakistan

Email address: hafizaanisaahmed@gmail.com

\section{Abstract}

The expeditious growth of the World Wide Web and the rampant flow of network traffic have resulted in a continuous increase of network security threats. Cyber attackers seek to exploit vulnerabilities in network architecture to steal valuable information or disrupt computer resources. Network Intrusion Detection System (NIDS) is used to effectively detect various attacks, thus providing timely protection to network resources from these attacks. To implement NIDS, stream of supervised and unsupervised machine learning approaches is applied to detect irregularities in network traffic and to address network security issues. Such NIDSs are trained using various datasets that include attack traces. However, due to the advancement in modern-day attacks, these systems are unable to detect the emerging threats. Therefore, NIDS needs to be trained and developed with a modern comprehensive dataset which contains contemporary common and attack activities. This paper presents a framework in which different machine learning classification schemes are employed to detect various types of network attack categories. Five machine learning algorithms: Random Forest, Decision Tree, Logistic Regression, K-Nearest Neighbors and Artificial Neural Networks, are used for attack detection. This study uses a dataset published by the University of New South Wales (UNSW-NB15), a relatively new dataset that contains a large amount of network traffic data with nine categories of network attacks. The results show that the classification models achieved the highest accuracy of $89.29 \%$ by applying the Random Forest algorithm. Further improvement in the accuracy of classification models is observed when Synthetic Minority Oversampling Technique (SMOTE) is applied to address the class imbalance problem. After applying the SMOTE, the Random Forest classifier showed an accuracy of $95.1 \%$ with 24 selected features from the Principal Component Analysis method. 


\section{Introduction}

41 In today's developed and interconnected world, the number of networks and data security breaches

42

43

44

45

46

47

48

49

50

51

52

53

54

55

56

57

58

59

60

61

62

63

64

65

66

67

68

69

70

71

72

73

74

75

76

77

78

79 is increasing immensely. The reasons include the growth of network traffic and advances in technology that have led to the creation of newer types of attacks. As a result, the level of attack eventually increases (Mikhail, Fossaceca and Iammartino, 2019).

There exist numerous network security attacks in today's era and to timely detect these attacks, several NIDSs are being developed and deployed. These NIDSs are widely used to protect digital resources against attacks and intrusions on networks (Zong, Chow and Susilo, 2018; Vidal and VidalMonge, 2019). Intrusion detection systems use two different methods, that is, anomaly-based detection and signature-based detection (Moustafa, Creech and Slay, 2017; Li et al., 2019). In an anomaly detection system, the network traffic is monitored and critical network characteristics are continuously tracked and analyzed (Habeeb et al., 2018). It generates alerts if unusual or anomalous activity is detected. Whereas, in signature detection system, well-known patterns of attacks (known as signatures) are stored. The network packets are searched for those patterns (Faker and Dogdu, 2019). If a pattern is accurately matched, the system generates an alert regarding that malicious activity (Azeez et al., 2020).

Although various attack detection mechanisms are available, they are still not effective enough to detect and analyze intrusions or malicious activities (Ahmed, Mahmood and Hu, 2016). Typically, anomaly-based detection systems are developed using different machine learning techniques for predicting intrusions in networks. Research has been conducted in this regard using datasets such as KDDCUP99 (Choudhary and Kesswani, 2020), KDD98 (W. Haider et al., 2017) and NSLKDD7 (Rathore and Park, 2018). However, due to the evolution of computer networks, these datasets are negatively affecting the results of NIDS (Khraisat et al., 2019). One of the factors influencing the results is the availability of modern-day attack data, as these datasets were created almost two decades ago. Consequently, due to revolution of network traffic, the traffic data available in those datasets is different from the existing modern-day traffic (Moustafa and Slay, 2016).

Improving the performance of existing NIDS requires modern and state-of-the-art datasets that are up-to-date. Therefore, a more efficient and more accurate evaluation of NIDS requires relatively new state-of-the-art datasets, including modern-day network's normal and attack activities. In this research, a framework has been developed for attack detection in a network using the UNSWNB15 dataset (Tama and Rhee, 2019; Aissa, Guerroumi and Derhab, 2020). This dataset is more recent and includes new attacks. Previously, KDDCUP99, KDD98, and NSL-KDD7 were widely used for NIDS benchmarked datasets. However, these older datasets are not as useful for today's network traffic (Viet et al., 2018; Kumar et al., 2020). However, few researchers have used the new data set, the UNSW-NB15 dataset, to detect an attack but their work has been limited (Bagui et al., 2019).

Peer] Comput. Sci. reviewing PDF | (CS-2021:09:65380:1:0:NEW 5 Nov 2021) 
80 The model proposed in this study uses the UNSW-NB15 1 dataset and not only achieves better

81

82

83

84

85

86

87

88

89

90

91

92

93

94

95

96

97

98

99

100

101

102

103

104

105

106

107

108

109

110

111

112

113

114

115

116

117

accuracy than previous research (Kasongo and Sun, 2020; Kumar, Das and Sinha, 2021) but also effectively detects all categories of attack. The work has been done using Python. Initially, preprocessing techniques were used on 80,000 randomly selected instances from the UNSW-NB15 dataset to normalize data values. Later, feature engineering has been performed to select the relevant features. To improve the performance of the classifiers, the research solved the problem of class imbalance using SMOTE. Subsequently, Random Forest (RF), Decision Tree (DT), Logistic Regression (LR), K-Nearest Neighbors (KNN) and Artificial Neural Network (ANN) have been used for classification. Lastly, evaluation metrics were used to compare the performance of all classifiers.

Following are the major contributions of this research:

- The dataset includes 45 features from which we identified 24 features that were most significant in identifying the attack.

- The various pre-processing techniques have been collectively applied to the UNSW-NB15 dataset to make the data meaningful and informative for model training.

- The class imbalance problem is addressed using when Synthetic Minority Oversampling Technique (SMOTE), thereby improving the detection rate of rare attacks.

- We have provided a comparison of five machine learning algorithms for detecting network attack categories.

The rest of the paper is organized as follows: In Section 2, related work has been presented; Section 3 describes the methodology of the framework developed; Section 4 and 5 elaborates the discussion of the experimental results and the last Section 6 hence concludes the paper.

\section{Related Work}

As technology advances with modern techniques, computer networks are using the latest technologies to put it into practice, which has dramatically changed the level of attacks. Therefore, to target the present-day attack categories, UNSW-NB15 dataset has been created (Moustafa and Slay, 2015; Viet et al., 2018)

The research conducted using the UNSW-NB15 dataset is still not sufficient. However, some of the research work done using datasets is discussed below. Table 1 presents the summary and comparison of the discussed related work.

Moustafa and Slay (Moustafa and Slay, 2015) developed a model that focused on the classification of attack families available in the UNSW-NB15 dataset. The study used the Association Rule Mining technique for feature selection. For classification, Expectation-Maximization (EM)

${ }^{1}$ https://research.unsw.edu.au/projects/unsw-nb15-dataset

Peer] Comput. Sci. reviewing PDF | (CS-2021:09:65380:1:0:NEW 5 Nov 2021) 
118 algorithm and NB have been used. However, the accuracy of both algorithms for detecting rare 119 attacks was not significantly higher as the Naïve Bayes had an accuracy of $78.06 \%$ and the 120 accuracy of EM was $58.88 \%$.

121

122

123

124

125

126

127

128

129

130

131

132

133

134

135

136

137

138

139

140

141

142

143

144

145

146

147

148

149

150

151

152

153

154

155

156

157

Moustafa and Slay (Moustafa and Slay, 2016) further extended their work in 2016 and used correlation coefficient and gain ratio for feature selection in their work. Thereafter, five classification algorithms of NB, DT, ANN, LR, and EM were used on the UNSW-NB15. Results showed that $85 \%$ accuracy was achieved using DT with 15.75 False Alarm Rate (FAR). This research utilized a subset of UNSW-NB15; however, detection accuracy was not satisfactory.

For detecting botnets and their tracks, Koroniotis et al. (Koroniotis et al., 2017) presented a framework using machine learning techniques on a subset of the UNSW-NB15 dataset using network flow identifiers. Four classification algorithms were used i.e., Association Rule Mining (ARM), ANN, NB and DT. The results showed that the DT obtained the highest accuracy of 93.23\% with a False Positive Rate (FPR) of $6.77 \%$.

In 2019, Meftah et al. (Meftah, Rachidi and Assem, 2019) applied a two-stage anomaly-based NIDS approach to detect network attacks. The proposed method used LR, Gradient Boost Machine (GBM) and Support Vector Machine (SVM) with the Recursive Feature Elimination (RFE) and RF feature selection techniques on a complete UNSW-NB15 dataset. The results showed that the accuracy of multi-classifiers using DT was approximately $86.04 \%$, respectively.

Kumar et al. (Kumar et al., 2020) proposed an integrated calcification-based NIDS using DT models with a combination of clusters created using the k-mean algorithm and IG's feature selection technique. The research utilized only 22 features and five types of network attacks of UNSW-NB15 dataset, and the RTNITP18 dataset, which served as a test dataset to test the performance of the proposed model. The result showed an accuracy of $84.83 \%$ using the proposed model and $90.74 \%$ using the C5 model of DT.

Kasongo and Sun (Kasongo and Sun, 2020) presented the NIDS approach using five classification algorithms of LR, KNN, ANN, DT and SVM in conjunction with the feature selection technique of the XGBoost algorithm. The research used the UNSW-NB15 dataset to apply binary and multiclass classification methods. Although binary classification performed well with an accuracy of $96.76 \%$ using the KNN classifier, multiclass classification didn't perform well as it achieved the highest accuracy of $82.66 \%$.

Kumar et al. (Kumar, Das and Sinha, 2021) proposed Unified Intrusion Detection System (UIDS) to detect normal traffic and four types of network attack categories by utilizing UNSW-NB15 dataset. Proposed UIDS model was designed with the set of rules $(R)$ derived from various DT models including k-means clustering and IG's feature selection technique. In addition, various

Peer] Comput. Sci. reviewing PDF | (CS-2021:09:65380:1:0:NEW 5 Nov 2021) 
158

159

160

161

162

163

164

165

166

167

168

169

170

171

172

173

174

175

176

177

178

179

180

181

182

183

184

185

186

187

188

189

190

191

192

193

194

195

196

197

algorithms such as C5, Neural Network and SVM were also used to train the model. As a result, the proposed model improved with an accuracy of $88.92 \%$ over other approaches. However, other algorithms such as C5, Neural Network and SVM achieved an accuracy of $89.76 \%, 86.7 \%$ and $78.77 \%$, respectively.

From a brief review of related literature as shown in Table 1, it is evident that more work needs to be done to identify the features for the families of network attacks. There is a need to determine a generic model that provides better accuracy for all the attacks presented in the dataset.

This research provides a model that determines a common subset of features. Subsequently, by using that feature subset we would be able to identify all attacks, belonging to any category with consistent accuracy. It focuses on the implementation of a generic model that provides improved classification accuracy. Moreover, there is limited research that has used the class imbalance technique to balance instances of rare attacks present in the dataset.

\section{Proposed Methodology}

The framework utilizes a subset of the UNSW-NB15 dataset. It consists of two main steps. The first step involves data pre-processing, in which standardization and normalization of data are performed. Due to the high dimensional nature of the dataset, some features that are irrelevant or redundant may lead to reduce the accuracy of attack detection. To solve this problem, feature selection is used, in which only the relevant subset of features is selected to eliminate useless and noisy features from multidimensional datasets. Afterward, we have then addressed the class imbalance problem. In the next step, different classifiers are trained with relevant features to detect all categories of attack to get maximum accuracy. Finally, accuracy, precision, recall and F1-score performance measures are used to evaluate the model. The proposed methodology that represents the overall framework is shown in Fig. 1.

\section{Dataset}

UNSW-NB15 dataset has been created by researchers in 2015 focusing on advanced network intrusion techniques. It contains 2.5 million records with 49 features (Dahiya and Srivastava, 2018). There are nine different classes of attack families with two label values i.e., normal or attack (Khan et al., 2019; Khammassi and Krichen, 2020) in the UNSW-NB15 dataset (Benmessahel, Xie and Chellal, 2018). These classes are described in Table 2.

\section{Dataset Pre-Processing}

This phase involves the following steps: data standardization and data normalization.

- Data Standardization

As there were features with different ranges of values in the dataset. Initially, we performed data standardization to convert the data from normal distribution into standard normal distribution. 
198 Therefore, after rescaling, a mean value of an attribute is equal to 0 and the resulting distribution

199

200

201

202

203

204

205

206

207

208

209

210

211

212

213

214

215

216

217

218

219

220

221

222

223

224

225

226

227

228

229

230

231

232

233

234

235

236

is equal to the standard deviation. The formula to calculate a standard score (z-score) is:

$$
z=\frac{(x-\mu)}{\sigma}
$$

Where $\mathrm{x}$ is the data sample, $\mu$ is the mean and $\sigma$ is the standard deviation (Xiao et al., 2019).

- Data Normalization

In data normalization, the value of each continuous attribute is scaled between 0 and 1 such that the result of attributes does not dominate each other (Gupta et al., 2016). In this research, the normalizer class of Python has been used. This class enables the normalization of a particular dataset.

\section{Feature Selection}

Feature selection is a technique that is used to select features that mostly correlate and contribute to the target variable of the dataset (Aljawarneh, Aldwairi and Yassein, 2017). In this research, feature selection is done using Correlation Attribute Evaluation (CA), Information Gain (IG) and Principal Component Analysis (PCA). CA measures the relationship between each feature with the target variable and select only those relative features that have moderately higher positive or negative values, i.e., closer to 1 or -1 (Sugianela and Ahmad, 2020). While IG feature selection technique is used to determine relevant features and minimizing noise caused by unrelated features. These relevant features are calculated from the entropy matrix which measures the uncertainty of the dataset (Kurniabudi et al., 2020). Through Principal Component Analysis, the size of large datasets is reduced by retaining the relevant features that depend on the target class (Kumar, Glisson and Benton, 2020).

The above-mentioned feature selection techniques help to train the model correctly with only the relevant features that accurately predict the target class.

\section{Class Imbalance}

The UNSW-NB15 dataset is highly imbalanced not only because the number of normal traffic instances is much higher than different attack categories, but also because the different categories of attack instances are not equal in distribution. This problem is known as "Class Imbalance".

Table 3 depicts the distribution of nine categories of attack and normal instances in the training dataset. The attack categories such as Analysis, Backdoor, Shellcode and Worms have very few instances. This highly imbalanced nature of the dataset causes problems in training machine learning algorithms for accurately predicting cyber-attacks. To address the class imbalance issue, this research uses SMOTE. SMOTE synthesizes instances of minority classes to balance all the 
237 classes in the dataset (Laureano, Sison and Medina, 2019). Table 4 shows the instance percentages

238 in each class after applying SMOTE.

239

240 Classification Algorithms

241 Five classification algorithms, that is, RF, DT, LR, KNN and ANN were employed to train the

242 model.

243

244 - Random Forest

245 Random Forest is an ensemble classifier that is used for improving classification results. It 246 comprises multiple Decision Trees. In comparison with other classifiers, RF provides lower 247 classification errors. Randomization is applied for the selection of the best nodes for splitting when 248 creating separate trees in RF (Jiang et al., 2018).

249

250

- Decision Tree

251

In the Decision Tree algorithm, the attributes are tested on internal nodes, the outcomes of the tests

252 are represented by branches, and leaf nodes hold labels of the classes (Afraei, Shahriar and Madani,

253 2019). Attribute selection methods are used for identifying nodes. Those selected attributes

254

255 minimize the information that is required for tuple classification in the resulted partition. Hence, reflecting the minimum uncertainty or impurity in those partitions. Therefore, minimizing the projected number of tests required for tuple classification. In this research, ID3 algorithms utilize entropy class to determine which attributes should be queried on, at every node of those decision trees.

- Logistic Regression

261

262

Logistic Regression is a probabilistic classification model. It casts the problem into a generalized linear regression form. It has a sigmoid curve. The equation of the sigmoid function or logistic 263

264 function is:

\section{5}

266

This function is used for mapping values to probabilities. It works by mapping real values to other

267 values between 0 and 1 (Kyurkchiev and Markov, 2016).

268

269

- K-Nearest Neighbors

270

In K-Nearest Neighbors, a new data point is attached with the data points in the training set and

271 based on that attachment, a value is assigned to that new data point. This uses feature similarity 272 for prediction. In KNN Euclidean, Manhattan or Hamming distance are used for calculating the 273 distance between a test data and each record of training data (Jain, Jain and Vishwakarma, 2020). 274 Afterward, according to the value of distance, the rows are sorted. From those rows, K rows from 
275 the top are selected. Based on the most frequent classes of these rows classes to the test points are 276 assigned.

277

278

- Artificial Neural Network

279 In the Artificial Neural Network algorithm, there are three layers that consist of computational units called neurons. These layers are input, output and hidden layers. The number of neurons in these layers depends on the features of the dataset and classes which have to be detected and chosen with different techniques. Different types of activation functions are used in the ANN algorithm for calculating the weighted sum of the connections between neurons. This algorithm has biases in the hidden layer and an output layer which are adjusted to reduce errors and improve accuracy in training and testing the model (Andropov et al., 2017).

286

287

288

289

290

291

292

293

294

295

296

297

298

299

300

301

302

303

304

305

306

307

308

Precision shows which portion of relevant instances is actually positive among the selected instances (Roy and Cheung, 2018). The following formula is used to calculate precision:

\section{Evaluation Metrics}

A confusion matrix is used for the comparison of the performance of machine learning algorithms. This matrix is used for the creation of different metrics by the combination of the values of True Negative (TN), True Positive (TP), False Negative (FN) and False Positive (FP) (Tripathy, Agrawal and Rath, 2016). Below are some of the performance measures to evaluate models by the use of the confusion matrix.

Accuracy shows the correctness or closeness of the approximated value to the actual or true value of the model which means a portion of the total samples that are classified correctly (Lin, Ye and $\mathrm{Xu}, 2019)$. The following formula is used to calculate the accuracy of the model:

$$
\text { Accuracy }=\frac{T P+T N}{T P+T N+F N+F P}
$$

Precision $=\frac{T P}{T P+F P}$

Recall or True Positive Rate (TPR) calculates the fraction of actual positives that are correctly identified (Ludwig, 2017). The formula used to find recall is:

\section{8}

F1-score is interpreted as the harmonic mean of precision and recall means it combines the calculate F1-score: 


$$
F 1=2 * \frac{\text { Precision } * \text { Recall }}{\text { Precision }+ \text { Recall }}
$$

314

\section{Experiment and Result Analysis}

316

317

318

319

320

321

322

323

324

325

326

327

328

329

330

331

332

333

334

335

336

337

338

339

340

341

342

343

344

345

346

347

348

349

Following the methodology depicted in Fig. 1, experimental setup is established. In this research, a sample of 80000 instances is randomly selected from the UNSW-NB15 dataset. Initially, data standardization and normalization have been performed to rescale data values of the dataset and then three feature selection techniques are applied to select the most relevant features. Afterward, the class imbalance problem is resolved using SMOTE. Lastly, five classification algorithms i.e., RF, DT, LR, KNN and ANN are used to classify between the attack categories and normal traffic.

\section{Performance Analysis of Classification Models without Feature Selection}

Table 5 summarizes the results of classification models without feature selection, in which RF achieved the highest accuracy, precision, recall, and F1-score values approximately $89.29 \%$, $76.9 \%, 72.6 \%$ and $74.1 \%$ respectively. Whereas, LR obtained the lowest accuracy, precision, recall, and F1-score values of approximately $82.07 \%, 50.5 \%, 42.6 \%$ and $42.3 \%$. DT achieved an accuracy of $88.29 \%$ with $70.5 \%$ precision, $72.7 \%$ recall and $71.4 \%$ F1-score. KNN also recorded the same accuracy as LR but with better precision, recall and F1-score results. Whereas, ANN recorded average performance with accuracy, precision, recall, and F1-score values of approximately $84.80 \%, 61.0 \%, 53.2 \%$ and $54.5 \%$.

\section{Performance Analysis of Classification Models with Feature Selection}

Three feature selection techniques i.e., CA, IG and PCA are used in this research.

The following 35 features are selected by applying IG: sttl, ct_state_ttl, ct_flw_http_mthd, sbytes, id, smean, sload, dur, sinpkt, rate, proto, ct_dst_src_ltm, service, dbytes, sjit, ct_srv_dst, dload, dinpkt, dmean, ct_srv_src, synack, tcprtt, ct_dst_sport_ltm, djit, ct_src_dport_ltm, dtcpb, stcpb, spkts, dloss, ct_dst_ltm, ackdat, label, dpkts, ct_src_ltm, sloss.

After applying CA method, 24 features have been achieved from the set of 49 features: id, ct_dst_sport_ltm, ct_dst_src_ltm, ct_src_dport_ltm, sttl, ct_srv_dst, ct_srv_src, ct_dst_ltm, ct_src_ltm, ct_state_ttl, state, swin, dwin, proto, service, rate, dttl, stcpb, dtcpb, dmean, dload, tcprtt, ackdat, synack.

By applying PCA, 151 subsets from the set of 49 features were resulted, out of which 10 subsets with $80 \%$ results were selected. After tremendous analysis and evaluation of these 10 subsets, 24 features have been extracted. Afterward, five classifiers were trained by using these features: id, dur, dwin, proto, djit, swin, smean, state, service, ct_src_dport_ltm, dbytes, ct_dst_ltm, 
350

351

352

353

354

355

356

357

358

359

360

361

362

363

364

365

366

367

368

369

370

371

372

373

374

375

376

377

378

379

380

381

382

383

384

385

386

387

388

389

ct_dst_sport_ltm, ct_src_ltm, dloss, ct_flw_http_mthd, ct_srv_dst, dpkts, sttl, dmean, spkts, sbytes, sloss and sinpkt.

After training the classifiers with the above features, the results showing in Table 6 were obtained.

It is observed using IG technique, that the RF classifier achieved the highest accuracy of $89.5 \%$ approx. with precision rate $(76.8 \%)$, recall $(72.3 \%)$ and F1-score $(73.7 \%)$. In contrast to other classifiers, LR and KNN didn't perform well with IG as their recall and F1-score have below 50\% scores. There is no much difference between the accuracy of RF and DT classifiers as both give almost the same accuracy, recall and F1-score measures using IG technique. The only difference is in the precision rate as RF achieved $76.8 \%$ and DT scored $69.6 \%$ precision value.

It is observed that the accuracy of all the classifiers decreased when the model is trained using the CA technique. RF classifier achieved the highest accuracy of $86.3 \%$ but with low precision, recall and F1-score measures. The accuracy of DT and ANN classifiers are approximately the same as the RF classifier with a minor difference of $2 \%$ to $5 \%$. However, ANN classifier has very lowperformance measures as compared to RF and DT. Also, LR and KNN have the lowest accuracy measures with poor performance metrics.

It is observed using PCA feature selection technique, that RF classifier obtained the highest accuracy of $89.3 \%$ with precision $(77.3 \%)$, recall $(70.8 \%)$ and F1-score $(73.1 \%)$ rates. All the classifiers achieved the accuracy in between $80 \%$ to $89 \%$ but with low performance measures as compared to IG feature selection technique. LR recorded the lowest recall rate with $40.6 \%$ and F1score with $40 \%$.

After evaluation of the performance of three feature selection methods, it was observed that the feature selection technique of IG and PCA performed well as compared to CA. RF and DT classifiers approximately achieved the same accuracy between $88 \%$ to $89 \%$ when trained with IG and PCA. However, for precision, recall and F1-score measures, these classifiers showed average scores. Therefore, it is concluded that, no major changes have been observed in the results after applying feature selection techniques as classifiers achieved almost same accuracy before feature selection.

\section{Performance Analysis of Classification Models by handling Imbalanced Data}

To handle imbalanced data, SMOTE technique has been applied in this research to adjust the class distribution of dataset and increase the instances of minority classes of those network attacks that has lower instances. After handling imbalance data, the results showing in Table 7 were obtained.

By using IG feature selection technique after applying SMOTE, it is observed that the RF classifier achieved highest accuracy of $95.1 \%$ with highest precision rate $(94.8 \%)$, recall $(95.7 \%)$ and F1- 
390

391

392

393

394

395

396

397

398

399

400

401

402

403

404

405

406

407

408

409

410

411

412

413

414

415

416

417

418

419

420

421

422

423

424

425

426

427

428

429

score (95.1\%). Also, the accuracy of DT is $94.7 \%$, almost nearest from the RF. The accuracy of both algorithms increased after handling imbalanced classes i.e., from $89.5 \%$ to $95.0 \%$ in $\mathrm{RF}$ and $88.5 \%$ to $94.5 \%$ in DT. Whereas, after applying SMOTE, LR and ANN didn't perform well as their accuracies were decreased from $82.2 \%$ to $69.4 \%$ in LR and $85.7 \%$ to $77.3 \%$ in ANN using IG method. The accuracy of KNN is almost the same using all three feature selection techniques but with good precision, recall and F1-score measures.

By using CA feature selection technique after applying SMOTE, it is noticed that RF and DT classifiers achieved highest accuracy in between $92.6 \%$ to $93.5 \%$ with above $90 \%$ precision, recall and F1-score measures. The accuracy of both the algorithms increased after applying SMOTE. Also, a minor change occurred in $\mathrm{KNN}$ as their accuracy is improved from $76.8 \%$ to $78.4 \%$ after handing imbalanced classes. However, when comes to LR and ANN, both algorithms did not perform well with class balance as their accuracies have been decreased i.e., in LR, from $74.5 \%$ to $62.0 \%$ and in ANN, from $80.3 \%$ to $71.7 \%$.

By using PCA after applying SMOTE, it is noticed that the RF classifier achieved the highest accuracy of $95.1 \%$ with a precision rate of $94.8 \%$, recall of $95.7 \%$ and $\mathrm{F} 1$-score of $95.1 \%$. DT classifier achieved the accuracy of $94.7 \%$, which is almost nearest to the accuracy of RF. After applying SMOTE, there is no change in the results of KNN using PCA method. However, the accuracy of LR and ANN decreased from $80.4 \%$ to $68.2 \%$ in LR and $85.2 \%$ to $77.6 \%$ in ANN but with increased precision, recall and F1-score measures.

\section{Overall Performance Evaluation of Classification Models after handling Class Imbalance}

After handling class balancing by using SMOTE, it is concluded that RF classifier performed well with good results up to $95.1 \%$ by using PCA feature selection technique. Also, it is noticed that class balancing did not impact on LR and ANN classifiers as their accuracy decreased after handling minority classes.

- Confusion Metrics of best performed Classifier: Random Forest

After analysis of the five classification models, it is observed that RF scheme provided the highest accuracy. On the basis of which, the confusion matrix of RF classification model is analyzed to observe the attack prediction accuracy of the nine categories of attacks separately.

In Fig. 2, it is depicted that all the normal traffic instances were identified correctly by RF (i.e., it had 100\% accuracy). In attack categories, all the instances of Backdoor, Shellcode and Worms were also identified correctly showing 100 prediction accuracy. Whereas, 1759 out of 1763 instances of Analysis attack (i.e., 99.77\% accuracy), 2341 out of 2534 instances of Fuzzers (i.e., 92.38\% accuracy), 5461 out of 5545 instances of Generic (i.e., $98.49 \%$ accuracy), 2151 out of 2357 instances of Reconnaissance (i.e., $91.26 \%$ accuracy) were identified correctly. 
430

431

\section{Discussion}

433

434

435

436

437

438

439

440

441

442

443

444

445

446

447

448

449

450

451

452

453

454

455

456

457

458

459

460

461

462

463

464

465

466

467

468

\section{Conclusions}

The research proposed a framework that predicts a variety of network attack categories using supervised machine learning algorithms. The dataset used in this study is the UNSW-NB15 dataset, a relatively new and containing a large amount of network traffic data, with nine types of network attack categories.

The proposed framework implies five machine learning algorithms in conjunction with preprocessing techniques, different methods of feature selection and SMOTE. After training, the results of the classifiers shown in Table 6,7 and 8 were obtained.

Compared to previous studies, as shown in Table 1, our model performed well with the highest accuracy of $95.1 \%$ using RF classifier with 24 features selected by PCA after applying SMOTE. DT classifier has also performed well with accuracy between $92.6 \%$ to $94.7 \%$ using different feature selection techniques. Existing studies that summarized in Table 1, achieved less than 90\% accuracy, except for the research proposed by Kumar et al. (Kumar et al., 2020) and Koroniotis et al. (Koroniotis et al., 2017). Kumar et al. showed 90.74\% accuracy using the C5 model of DT in conjunction with the IG feature selection technique. While the model proposed by Koroniotis et al. achieved 93.23\% accuracy using DT classifier. In addition, none of the studies listed in Table 1 have resolved the class imbalance problem of the UNSW-NB15 dataset as there are many studies (Al-Daweri et al., 2020; Ahmad et al., 2021; Bagui and Li, 2021; Dlamini and Fahim, 2021) that have highlighted this issue. We addressed the class imbalance problem by applying SMOTE that improved the performance of the classifiers and achieved good results.

This paper presents a framework for network intrusion detection. The performance of the proposed framework has been analyzed and evaluated on the UNSW-NB15 dataset. The proposed framework uses different pre-processing techniques that includes data standardization and normalization, feature selection techniques and class balancing methods. The usability of the selected features along with using data standardization and normalization techniques is analyzed by applying them on five different classification models. The results showed that the features selected by PCA contributed much to improve accuracy than other methods. For improving the accuracy of the classification models, the class imbalance problem is also addressed which increased the framework performance with high margin. In can be concluded on the basis of evaluation results that both RF and DT classifiers performed well over the UNSW-NB15 dataset in terms of accuracy, precision, recall, and F1-score metrics. It can also be concluded that major issue in UNSW-NB15 dataset is not only the presence of highly correlated features but also the class imbalance problem of the dataset. Therefore, we used a novel combination of different pre-

Peer] Comput. Sci. reviewing PDF | (CS-2021:09:65380:1:0:NEW 5 Nov 2021) 
469 processing techniques in order to resolve all the underlying issues of the dataset and developed a 470 fast and efficient network security intrusion detection system.

471

\section{References}

473 Afraei, S., Shahriar, K. and Madani, S. H. (2019) 'Developing intelligent classification models for 474 rock burst prediction after recognizing significant predictor variables, Section 2: Designing 475 classifiers', in Tunnelling and Underground Space Technology. Elsevier, pp. 522-537. doi: 476 https://doi.org/10.1016/j.tust.2018.09.022.

477 Ahmad, M. et al. (2021) 'Intrusion detection in internet of things using supervised machine 478 learning based on application and transport layer features using UNSW-NB15 data-set', Eurasip 479 Journal on Wireless Communications and Networking, (1). doi: 10.1186/s13638-021-01893-8.

480 Ahmed, M., Mahmood, A. N. and Hu, J. (2016) 'A survey of network anomaly detection 481 techniques', Journal of Network and Computer Applications, 60, pp. 19-31.

482 Aissa, N. B., Guerroumi, M. and Derhab, A. (2020) 'NSNAD : negative selection-based network anomaly detection approach with relevant feature subset', Neural Computing and Applications, 32(8), pp. 3475-3501. doi: https://doi.org/10.1007/s00521-019-04396-2.

485

486 Al-Daweri, M. S. et al. (2020) 'An analysis of the KDD99 and UNSW-NB15 datasets for the intrusion detection system', Symmetry, 12(10), pp. 1-32. doi: 10.3390/sym12101666.

Aljawarneh, S., Aldwairi, M. and Yassein, M. B. (2017) 'Anomaly-based intrusion detection system through feature selection analysis and building hybrid efficient model', Journal of Computational Science, 25, pp. 152-160. doi: https://doi.org/10.1016/j.jocs.2017.03.006.

490

491

492

493

494 Andropov, S. et al. (2017) 'Network Anomaly Detection using Artificial Neural Networks', in 2017 20th Conference of Open Innovations Association (FRUCT). Saint Petersburg, Russia: IEEE, pp. 26-31. doi: 10.23919/FRUCT.2017.8071288.

Azeez, N. A. et al. (2020) 'Intrusion Detection and Prevention Systems: An Updated Review', in Data Management, Analytics and Innovation. Springer Singapore, pp. 685-696. doi: https://doi.org/10.1007/978-981-32-9949-8_48.

Bagui, S. and Li, K. (2021) 'Resampling imb̄alanced data for network intrusion detection datasets', Journal of Big Data, 8(1). doi: 10.1186/s40537-020-00390-x.

498 Bagui, Sikha et al. (2019) 'Using machine learning techniques to identify rare cyber-attacks on the 499 UNSW-NB15 dataset', Security and Privacy, 2(6), pp. 1-13. doi: https://doi.org/10.1002/spy2.91. 500 Benmessahel, I., Xie, K. and Chellal, M. (2018) 'A new evolutionary neural networks based on

501

502

503

504

505 intrusion detection systems using multiverse optimization', Applied Intelligence, 48(8), pp. 23152327. doi: https://doi.org/10.1007/s10489-017-1085-y.

Choudhary, S. and Kesswani, N. (2020) 'Analysis of KDD-Cup'99, NSL-KDD and UNSW-NB15 Datasets using Deep Learning in IoT', Procedia Computer Science, 167(2019), pp. 1561-1573. doi: https://doi.org/10.1016/j.procs.2020.03.367.

506

507

508

Dahiya, P. and Srivastava, D. K. (2018) 'Network Intrusion Detection in Big Dataset Using Spark', Procedia Computer Science, 132, pp. 253-262. doi: https://doi.org/10.1016/j.procs.2018.05.169.

509

510 Dlamini, G. and Fahim, M. (2021) 'DGM: a data generative model to improve minority class presence in anomaly detection domain', Neural Computing and Applications, 33(20), pp. 1363513646. doi: 10.1007/s00521-021-05993-w.

511 Faker, O. and Dogdu, E. (2019) 'Intrusion Detection Using Big Data and Deep Learning 512 Techniques', in ACM SE '19: Proceedings of the 2019 ACM Southeast Conference. Kennesaw 513 GA, USA: ACM, pp. 86-93. doi: https://doi.org/10.1145/3299815.3314439. 
514 Gupta, D. et al. (2016) 'Network intrusion detection system using various data mining techniques', 515 in 2016 International Conference on Research Advances in Integrated Navigation Systems 516 (RAINS). Bangalore, India: IEEE, pp. 1-6. doi: https://doi.org/10.1109/RAINS.2016.7764418.

517 Habeeb, R. A. A. et al. (2018) 'Real-time big data processing for anomaly detection : A Survey', 518 International Journal of Information Management, 45, pp. 289-307. doi: 519 https://doi.org/10.1016/j.ijinfomgt.2018.08.006.

520 Jain, S., Jain, S. C. and Vishwakarma, S. (2020) 'Analysis and Prediction of Customers' Reviews 521 with Amazon Dataset on Products', in ICT Systems and Sustainability. Singapore: Springer, pp. 522 445-456. doi: https://doi.org/10.1007/978-981-15-0936-0_48.

523 Jiang, J. et al. (2018) 'RST-RF : A Hybrid Model based on Rough Set Theory and Random Forest 524 for Network Intrusion Detection', in ICCSP 2018: Proceedings of the 2nd International 525 Conference on Cryptography, Security and Privacy. Guiyang, China: ACM, pp. 77-81. doi: 526 https://doi.org/10.1145/3199478.3199489.

527 Kasongo, S. M. and Sun, Y. (2020) 'Performance Analysis of Intrusion Detection Systems Using 528 a Feature Selection Method on the UNSW-NB15 Dataset', Journal of Big Data, 7(1). doi: 529 10.1186/s40537-020-00379-6.

530 Khammassi, C. and Krichen, S. (2020) 'A NSGA2-LR Wrapper Approach for Feature Selection 531 in Network Intrusion Detection', Computer Networks, 172(March), p. 107183. doi: 532 https://doi.org/10.1016/j.comnet.2020.107183.

533 Khan, F. A. et al. (2019) 'A Novel Two-Stage Deep Learning Model for Efficient Network 534 Intrusion Detection', IEEE Access, 7, pp. 30373-30385. doi: 535 https://doi.org/10.1109/ACCESS.2019.2899721.

536 Khraisat, A. et al. (2019) 'Survey of intrusion detection systems : techniques, datasets and 537 challenges', Cybersecurity, 2(1), pp. 2-20. doi: https://doi.org/10.1186/s42400-019-0038-7.

538 Koroniotis, N. et al. (2017) 'Towards Developing Network forensic mechanism for Botnet 539 Activities in the IoT based on Machine Learning Techniques', in International Conference on 540 Mobile Networks and Management. Springer, Cham., pp. 30-44.

541 Kumar, A., Glisson, W. B. and Benton, R. (2020) 'Network attack detection using an unsupervised 542 machine learning algorithm', in Proceedings of the Annual Hawaii International Conference on 543 System Sciences, pp. 6496-6505. doi: 10.24251/hicss.2020.795.

544 Kumar, V. et al. (2020) 'An integrated rule based intrusion detection system : analysis on UNSW545 NB15 data set and the real time online dataset', Cluster Computing, 23, pp. 1-22. doi: 546 https://doi.org/10.1007/s10586-019-03008-x.

547 Kumar, V., Das, A. K. and Sinha, D. (2021) 'UIDS: a unified intrusion detection system for IoT 548 environment', Evolutionary Intelligence, 14(1), pp. 47-59. doi: 10.1007/s12065-019-00291-w.

549 Kurniabudi et al. (2020) 'CICIDS-2017 Dataset Feature Analysis with Information Gain for 550 Anomaly Detection', IEEE Access, 8, pp. 132911-132921. doi: 10.1109/ACCESS.2020.3009843. 551 Kyurkchiev, N. and Markov, S. (2016) 'On the Hausdorff distance between the Heaviside step 552 function and Verhulst logistic function', Journal of Mathematical Chemistry, 54(1), pp. 109-119. 553 doi: https://doi.org/10.1007/s10910-015-0552-0.

554 Laureano, L. B., Sison, A. M. and Medina, R. P. (2019) 'Handling Imbalanced Data through 555 Affinity Propagation and SMOTE', in ICCBD 2019: Proceedings of the 2nd International 556 Conference on Computing and Big Data. Taichung, Taiwan: ACM, pp. 22-26. doi: 557 https://doi.org/10.1145/3366650.3366665.

558 Li, W. et al. (2019) 'Designing Collaborative Blockchained Signature-based Intrusion Detection 559 in', Future Generation Computer Systems, 96, pp. 481-489. doi: 
560 https://doi.org/10.1016/j.future.2019.02.064.

561 Lin, P., Ye, K. and Xu, C. (2019) 'Dynamic Network Anomaly Detection System by Using Deep

562 Learning Techniques', in CLOUD 2019: International Conference on Cloud Computing. San

563 Diego, CA, USA: Springer, Cham, pp. 161-176. doi: 10.1007/978-3-030-23502-4.

564 Ludwig, S. A. (2017) 'Intrusion Detection of Multiple Attack Classes using a Deep Neural Net

565 Ensemble', in IEEE Symposium Series on Computational Intelligence (SSCI). Honolulu, HI, USA:

566 IEEE, pp. 1-7. doi: https://doi.org/10.1109/SSCI.2017.8280825.

567 Meftah, S., Rachidi, T. and Assem, N. (2019) 'Network based intrusion detection using the 568 UNSW-NB15 dataset', International Journal of Computing and Digital Systems, 8(5), pp. 477569 487. doi: 10.12785/ijcds/080505.

570 Mikhail, J. W., Fossaceca, J. M. and Iammartino, R. (2019) 'A Semi-Boosted Nested Model With 571 Sensitivity-Based Weighted Binarization for Multi-Domain Network Intrusion Detection', $A C M$ 572 Transactions on Intelligent Systems and Technology, 10(3), pp. 1-27. doi: 573 https://doi.org/10.1145/3313778.

574 Moustafa, N., Creech, G. and Slay, J. (2017) 'Anomaly Detection System using Beta Mixture 575 Models and Outlier Detection', in Progress in Computing, Analytics and Networking. Advances in 576 Intelligent Systems and Computing. Springer, Singapore, pp. 125-135. doi: 577 https://doi.org/10.1007/978-981-10-7871-2_13.

578 Moustafa, N. and Slay, J. (2015) 'The significant features of the UNSW-NB15 and the KDD99 579 data sets for Network Intrusion Detection Systems', in 4th international workshop on building 580 analysis datasets and gathering experience returns for security (BADGERS). IEEE, pp. 25-31.

581 Moustafa, N. and Slay, J. (2016) 'The evaluation of Network Anomaly Detection Systems: 582 Statistical analysis of the UNSW-NB15 data set and the comparison with the KDD99 data set', 583 Information Security Journal A Global Perspective, 25(1-3), pp. 18-31.

584 Niyaz, Q. et al. (2016) 'A Deep Learning Approach for Network Intrusion Detection System', in 585 Proceedings of the 9th EAI International Conference on Bio-inspired Information and 586 Communications Technologies (BIONETICS). New York, USA: ACM, pp. 21-26. doi: https://doi.org/10.4108/eai.3-12-2015.2262516.

589 framework for IoT', Applied Soft Computing Journal, 72, pp. 79-89. doi: 590 https://doi.org/10.1016/j.asoc.2018.05.049.

591 Roy, B. and Cheung, H. (2018) 'A Deep Learning Approach for Intrusion Detection in Internet of 592 Things using Bi-Directional Long Short-Term Memory Recurrent Neural Network.', in 28th 593 International Telecommunication Networks and Applications Conference (ITNAC). Sydney, 594 NSW, Australia: IEEE, pp. 1-6. doi: https://doi.org/10.1109/ATNAC.2018.8615294.

595 Sugianela, Y. and Ahmad, T. (2020) 'Pearson Correlation Attribute Evaluation-based Feature 596 Selection for Intrusion Detection System', in International Conference on Smart Technology and 597 Applications (ICoSTA). Surabaya, Indonesia: IEEE. 598 10.1109/ICoSTA48221.2020.1570613717.

599 Tama, B. A. and Rhee, K.-H. (2019) 'An in-depth experimental study of anomaly detection using 600 gradient boosted machine', Neural Computing and Applications, 31(4), pp. 955-965. doi: 601 https://doi.org/10.1007/s00521-017-3128-z.

602 Tripathy, A., Agrawal, A. and Rath, S. K. (2016) 'Classification of Sentiment Reviews using N603 gram Machine Learning Approach', Expert Systems With Applications, 57, pp. 117-126. doi: 604 https://doi.org/10.1016/j.eswa.2016.03.028Get rights and content.

605 Vidal, J. M. and VidalMonge, M. A. S. (2019) 'Adversarial Communication Networks Modeling 
606 for Intrusion Detection Strengthened against Mimicry', in ARES '19:Proceedings of the 14th 607 International Conference on Availability, Reliability and Security. Canterbury CA, United 608 Kingdom: ACM, pp. 1-6. doi: https://doi.org/10.1145/3339252.3340335.

609 Viet, H. N. et al. (2018) 'Using Deep Learning Model for Network Scanning Detection', in ICFET 610 '18: Proceedings of the 4th International Conference on Frontiers of Educational Technologies. 611 Moscow, Russia: ACM, pp. 117-121. doi: https://doi.org/10.1145/3233347.3233379.

612 W. Haider et al. (2017) 'Generating realistic intrusion detection system dataset based on fuzzy 613 qualitative modeling', Journal of Network and Computer Applications, 87, pp. 185-192. doi: 614 https://doi.org/10.1016/j.jnca.2017.03.018.

615 Xiao, Y. et al. (2019) 'An Intrusion Detection Model Based on Feature Reduction and 616 Convolutional Neural Networks', IEEE Access, 7, pp. 42210-42219. doi: 617 10.1109/ACCESS.2019.2904620.

618 Zong, W., Chow, Y.-W. and Susilo, W. (2018) 'A two-stage classifier approach for network 619 intrusion detection', in ISPEC 2018: International Conference on Information Security Practice 620 and Experience. Tokyo, Japan: Springer, Cham, pp. 329-340. doi: https://doi.org/10.1007/978-3621 319-99807-7_20.

622 
Figure 1

Overall framework for predicting network attack categories 


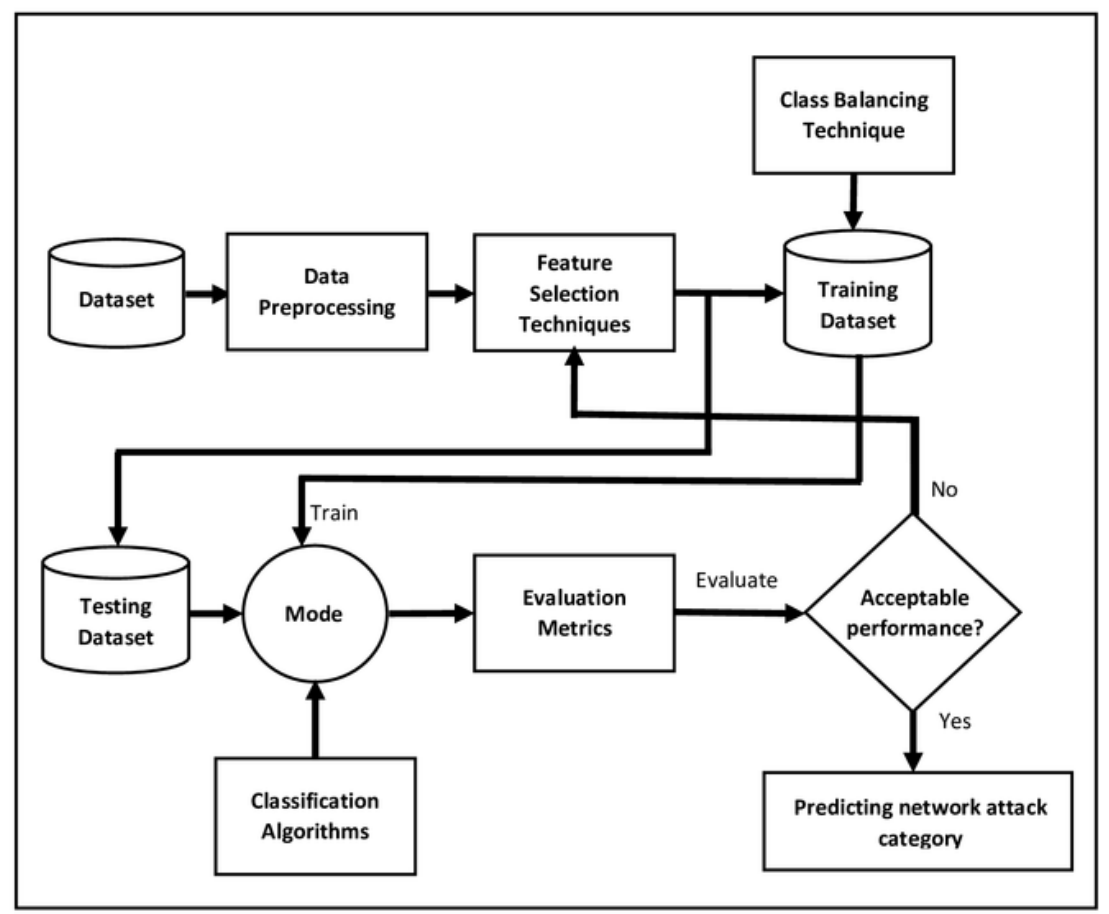

Overall framework for predicting network attack categories 
Figure 2

Confusion Matrix of Random Forest Classifier 


\begin{tabular}{|crrrrrrrrrrrr}
\hline \multicolumn{2}{r}{$\begin{array}{c}\text { Predicted } \\
\text { True }\end{array}$} & $\mathbf{0}$ & $\mathbf{1}$ & $\mathbf{2}$ & $\mathbf{3}$ & $\mathbf{4}$ & $\mathbf{5}$ & $\mathbf{6}$ & $\mathbf{7}$ & $\mathbf{8}$ & $\mathbf{9}$ \\
\hline Analysis & $\mathbf{0}$ & 1759 & 0 & 2 & 2 & 0 & 0 & 0 & 0 & 0 & 0 \\
Backdoor & $\mathbf{1}$ & 0 & 1762 & 0 & 0 & 0 & 0 & 0 & 0 & 0 & 0 \\
Dos & $\mathbf{2}$ & 39 & 33 & 3038 & 322 & 60 & 6 & 0 & 36 & 2 & 0 \\
Exploits & $\mathbf{3}$ & 51 & 33 & 424 & 5626 & 78 & 8 & 0 & 130 & 14 & 3 \\
Fuzzers & $\mathbf{4}$ & 10 & 13 & 56 & 78 & 2341 & 0 & 0 & 11 & 21 & 0 \\
Generic & $\mathbf{5}$ & 1 & 3 & 22 & 44 & 9 & 5461 & 0 & 0 & 0 & 0 \\
Normal & $\mathbf{6}$ & 0 & 0 & 0 & 0 & 0 & 0 & 7453 & 0 & 0 & 0 \\
Reconnaissance & $\mathbf{7}$ & 6 & 18 & 64 & 102 & 9 & 2 & 0 & 2151 & 0 & 0 \\
Shell code & $\mathbf{8}$ & 0 & 0 & 0 & 0 & 0 & 0 & 0 & 0 & 1651 & 0 \\
Worm & 9 & 0 & 0 & 0 & 0 & 0 & 0 & 0 & 0 & 0 & 1846 \\
& & & & & & & & & & & \\
\hline
\end{tabular}

Confusion Matrix of Random Forest Classifier 


\section{Table $\mathbf{1}$ (on next page)}

Summary of Existing Studies related to Network Attack Categories 
Table 1:

Summary of Existing Studies related to Network Attack Categories

\begin{tabular}{|c|c|c|c|c|}
\hline Reference & $\begin{array}{l}\text { Dataset } \\
\text { (complete } \\
\text { /Partial) }\end{array}$ & Algorithms & $\begin{array}{l}\text { Accuracy/ } \\
\text { FAR/FPR }\end{array}$ & Limitations \\
\hline $\begin{array}{l}\text { Moustafa } \\
\text { and Slay, } \\
2015\end{array}$ & $\begin{array}{l}\text { UNSW-NB15 } \\
\text { (Partial) } \\
\text { KDD99 } \\
\text { Dataset }\end{array}$ & $\begin{array}{l}\text { Naïve Bayes and } \\
\text { EM Algorithm }\end{array}$ & $\begin{array}{l}\text { Accuracy: } \\
\text { Naïve Bayes } \\
-37.5 \% \\
\text { EM } \\
\text { Algorithm: } \\
75.80 \% \\
\text { FPR: } 22.08\end{array}$ & $\begin{array}{l}\text { This research is } \\
\text { determining only five } \\
\text { network attack } \\
\text { categories. The problem } \\
\text { of class imbalance has } \\
\text { not been resolved. As a } \\
\text { result, the algorithms are } \\
\text { not performing well. }\end{array}$ \\
\hline $\begin{array}{l}\text { Moustafa } \\
\text { and Slay, } \\
2016\end{array}$ & $\begin{array}{l}\text { UNSW-NB15 } \\
\text { (Partial) } \\
\text { Dataset }\end{array}$ & $\begin{array}{l}\text { Naïve Bayes, } \\
\text { Decision Tree, } \\
\text { Artificial Neural } \\
\text { Network, Logistic } \\
\text { Regression, and } \\
\text { Expectation- } \\
\text { Maximisation }\end{array}$ & $\begin{array}{l}\text { Accuracy: } \\
\text { between } \\
78.47 \% \text { to } \\
85.56 \% \\
\text { FAR: } \\
\text { between } \\
15.75 \% \text { to } \\
23.79 \%\end{array}$ & $\begin{array}{l}\text { In this study, data pre- } \\
\text { processing techniques } \\
\text { have not been } \\
\text { implemented and the } \\
\text { issue of class imbalance } \\
\text { has not been resolved. }\end{array}$ \\
\hline $\begin{array}{l}\text { Koroniotis } \\
\text { et al., } 2017\end{array}$ & $\begin{array}{l}\text { UNSW-NB15 } \\
\text { Dataset }\end{array}$ & $\begin{array}{l}\text { Naive Bayes, } \\
\text { Decision Tree, } \\
\text { Association Rule } \\
\text { Mining (ARM) and } \\
\text { Artificial Neural } \\
\text { Network }\end{array}$ & $\begin{array}{l}\text { Accuracy: } \\
\text { between } \\
63.97 \% \text { to } \\
93.23 \% \\
\text { FPR: } \\
\text { between } \\
6.77 \% \text { to } \\
36.03 \%\end{array}$ & $\begin{array}{l}\text { This work didn't solve } \\
\text { the problem of class } \\
\text { imbalance. Hence, the } \\
\text { algorithms did not } \\
\text { perform well to detect } \\
\text { some network attacks. }\end{array}$ \\
\hline $\begin{array}{l}\text { Meftah, } \\
\text { Rachidi } \\
\text { and Assem, } \\
2019\end{array}$ & $\begin{array}{l}\text { UNSW-NB15 } \\
\text { Dataset }\end{array}$ & $\begin{array}{l}\text { Logistic } \\
\text { Regression, } \\
\text { Gradient Boost } \\
\text { Machine, and } \\
\text { Support Vector } \\
\text { Machine } \\
\end{array}$ & $\begin{array}{l}\text { Accuracy: } \\
\text { achieving a } \\
\text { multi- } \\
\text { classification } \\
\text { accuracy of } \\
86.04 \%\end{array}$ & $\begin{array}{l}\text { This research didn't } \\
\text { address the class } \\
\text { imbalance problem. }\end{array}$ \\
\hline $\begin{array}{l}\text { Kumar et } \\
\text { al., } 2020\end{array}$ & $\begin{array}{l}\text { UNSW-NB15 } \\
\text { (Partial) and } \\
\text { RTNITP18 } \\
\text { Dataset }\end{array}$ & $\begin{array}{l}\text { Decision Tree } \\
\text { Models (C5, } \\
\text { CHAID, CART, } \\
\text { QUEST) }\end{array}$ & $\begin{array}{l}\text { Accuracy: } \\
84.83 \% \\
\text { using } \\
\text { proposed } \\
\text { model and } \\
90.74 \% \\
\text { using C5 } \\
\text { model } \\
\end{array}$ & $\begin{array}{l}\text { This work has predicted } \\
\text { only } 4 \text { out of } 9 \text { categories } \\
\text { of the UNSW-NB15 } \\
\text { dataset. Also, the } \\
\text { problem of class } \\
\text { imbalance has not been } \\
\text { solved in this study. }\end{array}$ \\
\hline $\begin{array}{l}\text { Kasongo } \\
\text { and Sun, }\end{array}$ & $\begin{array}{l}\text { UNSW-NB15 } \\
\text { Dataset }\end{array}$ & $\begin{array}{l}\text { Logistic regression, } \\
\text { K-Nearest }\end{array}$ & $\begin{array}{l}\text { Accuracy: } \\
\text { between }\end{array}$ & $\begin{array}{l}\text { The problem of class } \\
\text { imbalance has not been }\end{array}$ \\
\hline
\end{tabular}




\begin{tabular}{|l|l|l|l|l|}
\hline 2020 & & $\begin{array}{l}\text { Neighbors, } \\
\text { Artificial Neural } \\
\text { Network, Decision } \\
\text { Tree and Support } \\
\text { Vector Machine }\end{array}$ & $\begin{array}{l}53.43 \% \text { to } \\
82.66 \% \\
\text { using } \\
\text { multiclass } \\
\text { classification } \\
\text { scheme }\end{array}$ & $\begin{array}{l}\text { resolved in this research. } \\
\text { Hence, the model has not } \\
\text { achieved good accuracy. }\end{array}$ \\
\hline $\begin{array}{l}\text { Kumar et } \\
\text { al., 2021 }\end{array}$ & $\begin{array}{l}\text { UNSW-NB15 } \\
\text { (Partial) } \\
\text { Dataset }\end{array}$ & $\begin{array}{l}\text { Decision Tree } \\
\text { Models (C5, } \\
\text { CHAID, CART, } \\
\text { QUEST) }\end{array}$ & $\begin{array}{l}\text { Accuracy: } \\
88.92 \% \\
\text { using } \\
\text { proposed } \\
\text { model }\end{array}$ & $\begin{array}{l}\text { Research has predicted } \\
\text { only 4 types of network } \\
\text { attack categories of the } \\
\text { UNSW-NB15 dataset. In } \\
\text { addition, the problem of } \\
\text { class imbalance has not } \\
\text { been resolved in this } \\
\text { research. }\end{array}$ \\
\hline
\end{tabular}




\section{Table 2 (on next page)}

Description of Network Attacks 
Table 1:

Description of Network Attacks

\begin{tabular}{|c|c|}
\hline Attack Family & Description \\
\hline Fuzzers & $\begin{array}{l}\text { These attacks attempt to crash servers on networks by inputting numerous } \\
\text { random data, called "Fuzz", in vulnerable points of the networks }\end{array}$ \\
\hline Analysis & $\begin{array}{l}\text { These attacks perform scanning of networks via ports (for example: port } \\
\text { scans, footprinting, vulnerability scans). }\end{array}$ \\
\hline Backdoors & $\begin{array}{l}\text { In this type of attacks intruder bypass normal authentication process of } \\
\text { system portals to get illegal access into system. These attacks use malicious } \\
\text { software's that provide remote access to the system to the attackers. }\end{array}$ \\
\hline $\begin{array}{l}\text { Denial of } \\
\text { service (DoS) }\end{array}$ & $\begin{array}{l}\text { In this type of attacks, the attackers send several illegal connection requests } \\
\text { to generate unwanted network traffic and make network services unavailable } \\
\text { for actual users. }\end{array}$ \\
\hline Exploits: & $\begin{array}{l}\text { In these attacks vulnerable points in the operating systems are targeted and } \\
\text { compromised. }\end{array}$ \\
\hline Generic & $\begin{array}{l}\text { This attack is a collision attack in which attackers tamper secret keys } \\
\text { generated by using cryptographic principles }\end{array}$ \\
\hline Reconnaissance & $\begin{array}{l}\text { These types of attacks try to find possible vulnerabilities in the computer } \\
\text { network and then further use different exploitation techniques to exploit a } \\
\text { compromised network. }\end{array}$ \\
\hline Shellcode & $\begin{array}{l}\text { These attacks comprise of a set of instructions that are used as a payload in } \\
\text { the exploitation of a certain network. These codes are inserted into software } \\
\text { to compromise and remotely access a computer system. }\end{array}$ \\
\hline Worms & $\begin{array}{l}\text { These are attacks are self-replicating malicious programs that exploit } \\
\text { computer systems by duplicating themselves to the uninfected computer } \\
\text { systems of the entire network system }\end{array}$ \\
\hline
\end{tabular}




\section{Table 3 (on next page)}

\section{Class Distribution in Dataset}


1 Table 1:

2 Class Distribution in Dataset

\begin{tabular}{|l|c|}
\hline Class Distribution & Instance count (\%) \\
\hline Normal & 32.10 \\
Generic & 22.80 \\
Exploits & 19.06 \\
Fuzzers & 10.32 \\
DoS & 7.08 \\
Reconnaissance & 5.85 \\
Analysis & 1.12 \\
Backdoor & 0.93 \\
Shellcode & 0.65 \\
Worms & 0.06 \\
\hline
\end{tabular}

3 


\section{Table 4 (on next page)}

Class Distribution in Dataset after applying SMOTE 
1 Table 1:

2 Class Distribution in Dataset after applying SMOTE

\begin{tabular}{|l|c|}
\hline Class Distribution & Instance count (\%) \\
\hline Normal & 22.13 \\
Generic & 18.31 \\
Exploits & 15.72 \\
Fuzzers & 10.05 \\
DoS & 7.11 \\
Reconnaissance & 6.62 \\
Analysis & 5.21 \\
Backdoor & 5.08 \\
Shellcode & 4.95 \\
Worms & 4.76 \\
\hline
\end{tabular}

3 


\section{Table 5 (on next page)}

Results of classification models without feature selection 
Table 1:

Results of classification models without feature selection

\begin{tabular}{|l|c|c|c|c|}
\hline \multicolumn{1}{|c|}{ Method } & Accuracy\% & Precision\% & Recall\% & F1-Score\% \\
\hline Random Forest & 89.29 & 76.9 & 72.6 & 74.1 \\
\hline Decision Tree & 88.29 & 70.5 & 72.7 & 71.4 \\
\hline Logistic Regression & 82.07 & 50.5 & 42.6 & 42.3 \\
\hline K-Nearest Neighbor & 82.37 & 52.5 & 47.6 & 49.1 \\
\hline Artificial Neural Network & 84.80 & 61.0 & 53.2 & 54.5 \\
\hline
\end{tabular}




\section{Table 6(on next page)}

Results of classification models with feature selection 
1 Table 1:

2 Results of classification models with feature selection

3

\begin{tabular}{|c|c|c|c|c|c|}
\hline Method & Feature Selection Techniques & $\begin{array}{c}\text { Accuracy } \\
\%\end{array}$ & $\begin{array}{c}\text { Precision } \\
\%\end{array}$ & $\begin{array}{c}\text { Recall } \\
\%\end{array}$ & $\begin{array}{c}\text { F1- } \\
\text { Score } \%\end{array}$ \\
\hline \multirow{3}{*}{$\begin{array}{l}\text { Random } \\
\text { Forest }\end{array}$} & Information Gain Attribute & 89.5 & 76.8 & 72.3 & 73.7 \\
\hline & Correlation Attribute & 86.3 & 72.2 & 59.6 & 61.9 \\
\hline & Principal Component Analysis & 89.3 & 77.3 & 70.8 & 73.1 \\
\hline \multirow{3}{*}{$\begin{array}{l}\text { Decision } \\
\text { Tree }\end{array}$} & Information Gain Attribute & 88.5 & 69.6 & 72.0 & 70.7 \\
\hline & Correlation Attribute & 84.8 & 63.3 & 60.9 & 61.9 \\
\hline & Principal Component Analysis & 88.4 & 70.9 & 67.3 & 69.3 \\
\hline \multirow{3}{*}{$\begin{array}{l}\text { Logistic } \\
\text { Regression }\end{array}$} & Information Gain Attribute & 82.2 & 51.2 & 42.3 & 41.9 \\
\hline & Correlation Attribute & 74.5 & 38.5 & 36.0 & 35.4 \\
\hline & Principal Component Analysis & 80.4 & 51.3 & 40.6 & 40.0 \\
\hline \multirow{3}{*}{$\begin{array}{l}\text { K-Nearest } \\
\text { Neighbor }\end{array}$} & Information Gain Attribute & 82.7 & 54.0 & 48.1 & 49.6 \\
\hline & Correlation Attribute & 76.8 & 46.3 & 42.6 & 43.5 \\
\hline & Principal Component Analysis & 84.0 & 57.8 & 51.3 & 53.3 \\
\hline \multirow{3}{*}{$\begin{array}{l}\text { Artificial } \\
\text { Neural } \\
\text { Network }\end{array}$} & Information Gain Attribute & 85.7 & 60.6 & 54.6 & 54.2 \\
\hline & Correlation Attribute & 80.3 & 50.1 & 44.1 & 44.7 \\
\hline & Principal Component Analysis & 85.2 & 61.2 & 52.4 & 54.4 \\
\hline
\end{tabular}




\section{Table 7 (on next page)}

Results of classification models with feature selection after handling Class Imbalance 
1 Table 1:

2 Results of classification models with feature selection after handling Class Imbalance

\begin{tabular}{|c|c|c|c|c|c|}
\hline Method & Feature Selection Techniques & $\begin{array}{c}\text { Accuracy } \\
\%\end{array}$ & $\begin{array}{c}\text { Precision } \\
\%\end{array}$ & $\begin{array}{c}\text { Recall } \\
\%\end{array}$ & $\begin{array}{c}\text { F1- } \\
\text { Score } \%\end{array}$ \\
\hline \multirow{3}{*}{$\begin{array}{l}\text { Random } \\
\text { Forest }\end{array}$} & Information Gain Attribute & 95.0 & 94.7 & 95.7 & 95.1 \\
\hline & Correlation Attribute & 93.5 & 93.0 & 94.2 & 93.5 \\
\hline & Principal Component Analysis & 95.1 & 94.8 & 95.7 & 95.1 \\
\hline \multirow{3}{*}{$\begin{array}{l}\text { Decision } \\
\text { Tree }\end{array}$} & Information Gain Attribute & 94.5 & 94.1 & 95.2 & 94.7 \\
\hline & Correlation Attribute & 92.6 & 91.8 & 93.4 & 92.6 \\
\hline & Principal Component Analysis & 94.7 & 94.4 & 95.4 & 94.8 \\
\hline \multirow{3}{*}{$\begin{array}{l}\text { Logistic } \\
\text { Regressio } \\
n\end{array}$} & Information Gain Attribute & 69.4 & 61.0 & 59.4 & 56.2 \\
\hline & Correlation Attribute & 62.0 & 48.1 & 50.6 & 45.8 \\
\hline & Principal Component Analysis & 68.2 & 57.8 & 58.0 & 54.8 \\
\hline \multirow{3}{*}{$\begin{array}{l}\text { K-Nearest } \\
\text { Neighbor }\end{array}$} & Information Gain Attribute & 82.7 & 79.4 & 82.6 & 80.6 \\
\hline & Correlation Attribute & 78.4 & 74.3 & 78.3 & 75.8 \\
\hline & Principal Component Analysis & 84.7 & 82.2 & 85.1 & 83.1 \\
\hline \multirow{3}{*}{$\begin{array}{l}\text { Artificial } \\
\text { Neural } \\
\text { Network }\end{array}$} & Information Gain Attribute & 77.3 & 75.0 & 70.5 & 71.0 \\
\hline & Correlation Attribute & 71.7 & 69.2 & 62.1 & 63.5 \\
\hline & Principal Component Analysis & 77.6 & 76.2 & 70.6 & 71.5 \\
\hline
\end{tabular}

